\title{
Fructo-oligosaccharides (FOS) production by fungal submerged culture using aguamiel as a low-cost by-product
}

\author{
Diana B. Muñiz-Márquez ${ }^{\mathrm{a}, \mathrm{b}}$, José A. Teixeira ${ }^{\mathrm{c}}$, Solange I. Mussatto ${ }^{\mathrm{d}}$, Juan C. Contreras-Esquivel ${ }^{\mathrm{a}}$, \\ Raúl Rodríguez-Herrera ${ }^{a}$, Cristóbal N. Aguilar ${ }^{\mathrm{a}, *}$
}

${ }^{a}$ Food Research Department, School of Chemistry, University Autonomous of Coahuila, 25280, Saltillo, Coahuila, Mexico

${ }^{\mathrm{b}}$ Departamento de Ingenierías, Tecnológico Nacional de México Campus Instituto Tecnológico de Ciudad Valles, 79010, Ciudad Valles, San Luis Potosí, Mexico

${ }^{\mathrm{c}}$ Centre of Biological Engineering, University of Minho, Campus Gualtar, 4710-057, Braga, Portugal

${ }^{\mathrm{d}}$ Novo Nordisk Foundation Center for Biosustainability, Technical University of Denmark, Kemitorvet, Building 220, 2800, Kongens Lyngby, Denmark

\section{A R T I C L E I N F O}

\section{Keywords:}

Aguamiel

Aspergillus

Submerged fermentation

Fructooligosaccharides (FOS)

\begin{abstract}
A B S T R A C T
Fructooligosaccharides (FOS) are important ingredients in the functional food industry because they have different biological properties such as decrease level of triglycerides, cholesterol and phospholipids and stimulate growth of probiotics for enhancement of microflora in large intestine. However, current strategies for the FOS production through simple and economical bioprocess has been necessary. The aim of this work was evaluated the capacity of three fungal strains (Aspergillus niger GH1, Aspergillus niger PSH and Aspergillus oryzae DIA-MF) to produce fructooligosaccharides (FOS) using aguamiel from Agave salmiana as an economical substrate. In addition, Czapek Dox medium supplemented with sucrose as carbon source was used as a control medium for the FOS production. A. oryzae DIA-MF was a fungi producer of FOS using aguamiel or Czapek Dox medium as substrate at $24 \mathrm{~h}$ of fermentation. However, the yield of FOS was increased two folds $(20.30 \mathrm{~g} / \mathrm{L})$, with a productivity of $0.84 \mathrm{~g}$ FOS/ 1 .h when aguamiel was used as substrate. On the other hand, A. niger GH1 and A. niger PSH showing only hydrolytic activity on sucrose under the studied conditions. In conclusion, this study shown excellent compatibility of $A$. oryzae DIA-MF using aguamiel as an economical substrate for the FOS production under a simple bioprocess.
\end{abstract}

\section{Introduction}

Fructooligosaccharides (FOS) are non-digestible ingredients that promote the grown and activity of beneficial microorganism called probiotics (lactobacilli and bifidobacteria) in the host intestine reducing the incidence of intestinal diseases. Structurallly, refer to a series of oligosaccharides composed of 1-kestose $\left(\mathrm{GF}_{2}\right)$, nystose $\left(\mathrm{GF}_{3}\right)$ and $1^{\mathrm{F}}-\beta$ fructofuranosyl nystose $\left(\mathrm{GF}_{4}\right)$ (Zhang et al., 2017). FOS are found in small quantities in several vegetal such as banana, asparagus, Jerusalem artichoke, onions, etc. For large scale production, microbial enzymes $\beta$-fructofuranosidases (FFases, EC.3.2.1.26) and fructosyltransferases (FTases EC.2.4.1.9) (Batista, Simões, Barros, Pessoni, Braga \& Ribeiro, 2013) are used. These enzymes have been found in filamentous fungi especially of the genus Aspergillus sp. Fusarium sp., Aerobasidium sp., Penicllum sp. and Arthrobacter sp. (Castro, Nobre, Duprez, Weireld \& Hanston, 2017; Xie, Zhou, Liu, Zhang, Li, Zhao, Sun \& Zhong, 2017). However, important aspects in submerged fermentation for the FOS production should be established for each microbial strain, for example high levels of sucrose (700-850 g.L) in the culture are preferred by these microorganisms, also, agitation, $\mathrm{pH}$, temperature and other nutritional requirements such as some minerals in the culture medium are necessary for the FOS synthesis (Won, 1996). But, other factors limiting their synthesis, for example the cost of enzyme production, their purification and the use of commercial culture medium (Nascimento, Nobre, Cavalcanti, Teixeira, \& Porto, 2016). Therefore, recent studies have been focused on use of low cost materials as a viable alternative for FOS production (Xie et al., 2017). Agave plants are abundant in some regions of Mexico and some species are known as "magueyes pulqueros" (Agave salmiana, agave mapisaga, A. atrovirens, A. americana, A. ferox) because their main use is for the pulque production. Pulque is a traditional Mexican alcoholic beverage produced by the natural fermentation of aguamiel (mead) that is a fresh sap obtained from the adult plant from 8 to 10 years old (mature maguey). The fermented product is a milk white viscous and acid liquid with alcohol content between 4 and $7^{\circ} \%$ vol. (Hernandez-López et al., 2018). Recent studies have reported high sucrose content in sterile aguamiel (Muñiz

\footnotetext{
* Corresponding author.

E-mail address: cristobal.aguilar@uadec.edu.mx (C.N. Aguilar).
} 
et al., 2015) and some functional properties such as prebiotic effect related with the presence of fructooligosaccharides have been reported (Hernandez-López et al., 2018). Therefore, the use of aguamiel for the FOS production may contribute to reduce production costs compared with the utilization of commercial culture media. Then, the objective of this work was evaluated the capacity of three fungal strains (Aspergillus niger GH1, Aspergillus niger PSH and Aspergillus oryzae DIA-MF) to produce fructooligosaccharides (FOS) under submerged culture using aguamiel from Agave salmiana as an alternative substrate.

\section{Materials and methods}

\subsection{Chemicals}

FOS standards 1-kestose $\left(\mathrm{GF}_{2}\right)$, 1-nystose $\left(\mathrm{GF}_{3}\right)$ and $1^{\mathrm{F}}$ - fructofuranosylnystose $\left(\mathrm{GF}_{4}\right)$ were purchased in Wako Pure Chemical Industries, Ltd. (Japan). Sugars fructose (F), glucose (G) and sucrose (S) were obtained from Sigma Aldrich (St. Louis, MO, USA). Aguamiel was collected from a maguey commercial plantation at the Mangas, Coahuila, México in February 2013.

\subsection{Microorganisms and inoculum preparation}

Three fungal strains Aspergillus niger (GH1, PSH) and Aspergillus oryzae (DIA-MF) were tested for fructooligosaccharides (FOS) production. The strains belonging to the Food Research Department-UAdeC collection were reactivated on potato dextrose agar (PDA) at $30 \pm 1{ }^{\circ} \mathrm{C}$ for 5 days. To prepare spores suspension, the fungal strains were inoculated in flasks containing PDA medium at $28 \pm 1{ }^{\circ} \mathrm{C}$ for 5 days. Then, spores were scraped down from flasks with a $0.01 \% \mathrm{w} / \mathrm{v}$ sterilized solution Tween 80. Spores suspension was adjusted to $1 \times 10^{7}$ spores $\mathrm{mL}^{-1}$ by a Neubauer chamber under sterile conditions (Castro, Nobre, Duprez, De Weirelda, \& Hantsona, 2017).

\subsection{Fermentation conditions}

Aguamiel from Agave salmiana was used as culture medium in the fermentation. Previously, was filtered, centrifuged at $8000 \mathrm{rpm}$ for $10 \mathrm{~min}$ at $20^{\circ} \mathrm{C}$ and sterilized at $112^{\circ} \mathrm{C}$ for $15 \mathrm{~min}$. Fermentation was performed in $10 \mathrm{~mL}$ glass reactors with aguamiel as a sole source of nutrients. The reactors were inoculated with a spore suspension containing $1 \times 10^{7}$ spores $\mathrm{mL}^{-1}$ and were incubated in a Shaking Incubator Sheldon Manufacturing, Inc. (SHEL LAB) at $30 \pm 1{ }^{\circ} \mathrm{C}$ and $180 \mathrm{rpm}$ for $120 \mathrm{~h}$. During fermentation process the samples were withdrawn at regular time intervals at $0,24,48,72,96$ and $120 \mathrm{~h}$ and were filtered using a filter paper (Whatman no. 1) and then centrifuged at $2500 \mathrm{rpm}$ for $5 \mathrm{~min}$. All experiments were carried out in duplicate.

\subsection{Comparative FOS production with Czapek Dox medium}

Czapek-Dox culture medium was used as synthetic medium for FOS production with the following composition: $\mathrm{NaNO}_{3} 0.25 \%, \mathrm{KH}_{2} \mathrm{PO}_{4}$ $0.1 \%, \mathrm{MgSO}_{4} 0.05 \%, \mathrm{KCl} 0.05 \%$ containing the same initial concentration of sugars (fructose, glucose and sucrose) that the aguamiel: 17.9, 14.4 and $42.6 \mathrm{~g} / \mathrm{L}$ respectively. Here, were used the same fermentation conditions described in section 2.3. All experiments were carried out in duplicate.

\subsection{FOS and residual sugars quantification}

Fermented extracts were filtered through a $0.45 \mu \mathrm{m}$ nylon membrane (Millipore) and were used for determination of FOS (1-kestose, 1nystose and $1^{\mathrm{F}}$ - fructofuranosylnystose), residual sugars, fructose, glucose, and sucrose. Samples were analyzed employing high performance liquid chromatography (HPLC, Perkin Elmer Series 200) with a Prevail Carbohydrate ES Column $(5 \mu \mathrm{m}, 250 \times 4.6 \mathrm{~mm}$, Grace $)$ at $30^{\circ} \mathrm{C}$. A mixture of acetonitrile/water 70:30 (v/v) was used as mobile phase at a flow rate of $1 \mathrm{~mL} / \mathrm{min}$ with a pressure of $1700 \mathrm{psi}$. A refractive index detector (RID) was operated at $35^{\circ} \mathrm{C}$. The response of the RID was recorded and integrated using the TOTALCHROM WS V6.3 software. The quantification of sugars and FOS in samples was determined a through standards curves made with known concentrations of each compound (Nobre et al., 2018).

\subsection{Maximization of the FOS production}

FOS production was maximized using $250 \mathrm{~mL}$ flasks containing $30 \mathrm{~mL}$ aguamiel (initial $\mathrm{pH} 4.5$ ) sterilized at $112^{\circ} \mathrm{C}$ during $15 \mathrm{~min}$. Aspergillus oryzae (DIA-MF) was tested as FOS-producer fungus. The flasks were inoculated with $1 \times 10^{7}$ fungal spores $\mathrm{mL}^{-1}$ and incubating at $30 \pm 1{ }^{\circ} \mathrm{C}$ until $144 \mathrm{~h}$ on rotatory shaking incubator (INNOVA 44) at $180 \mathrm{rpm}$. The samples were withdrawn at the follow time intervals: 0 , $12,18,24,42,48,72,96$ and $120 \mathrm{~h}$ of fermentation. All experiments were done in triplicate.

\subsection{Analysis of FOS and sugars in fermented extracts}

FOS and other sugars (fructose, glucose and sucrose) contents were obtained analyzing samples using a high-performance liquid chromatography (HPLC) with the analytic methodology described in section 2.5 (Nobre et al., 2018). Briefly, samples were filtered with nylon membranes $(0.45 \mu \mathrm{m})$ and analyzed using HPLC Perkin Elmer Series 200 system with a Prevail Carbohydrate ES Column $(5 \mu \mathrm{m}$, $250 \times 4.6 \mathrm{~mm}$, Grace) at $30^{\circ} \mathrm{C}$. A mixture of acetonitrile/water $70: 30$ $(v / v)$ was used as mobile phase at a flow rate of $1 \mathrm{~mL} / \mathrm{min}$ with a pressure of $1700 \mathrm{psi}$. A refractive index detector (RID) was operated at $35^{\circ} \mathrm{C}$. The response of the RID was recorded and integrated using the TOTALCHROM WS V6.3 software. The quantification of sugars and FOS in samples was performed using standards curves made with known concentrations of each compound.

\subsection{Fungal biomass quantification}

The fermented extract was transferred to conical tubes and centrifugated at $8000 \mathrm{rpm}$ at $15^{\circ} \mathrm{C}$ for $10 \mathrm{~min}$, then biomass was dried at $70{ }^{\circ} \mathrm{C}$ to constant weight. Biomass yield was calculated as dry cell weight per volume (g/L) (Ganaie, Gupta, \& Kango, 2013).

\subsection{Kinetic parameters calculation}

The total FOS was calculated by the sum of 1-kestose, 1-nystose and 1 - $\beta$-fructofuranosylnystose in $\mathrm{g} / \mathrm{L}$. The FOS yield ( $\mathrm{Y}_{\mathrm{P} / \mathrm{S}, \mathrm{g} / \mathrm{g}}$ ) was determined by the ratio between total FOS produced and consumed sucrose in $\mathrm{g} / \mathrm{L}$.

\subsection{Experimental design and analysis data}

A randomized design with two replications was employed for each case and data was analyzed using analysis of variance (ANOVA) employing the software SAS V 9.0. The Tukey test was used for the multiple comparison in each assay. The differences between mean values were considered significant at $\mathrm{p}<0.5$.

\section{Results and discussion}

\subsection{Selection of the microorganism}

When Aspergillus niger (GH1 and PSH) were used higher levels of glucose as by-product were observed during the fermentation, especially between the $24-30 \mathrm{~h}$. In the case of $A$. niger $\mathrm{GH} 1$ also fructose was increased, which indicate the production of hydrolases by both microorganisms which hydrolyze aguamiel sucrose (Fig. 1a-b). The 

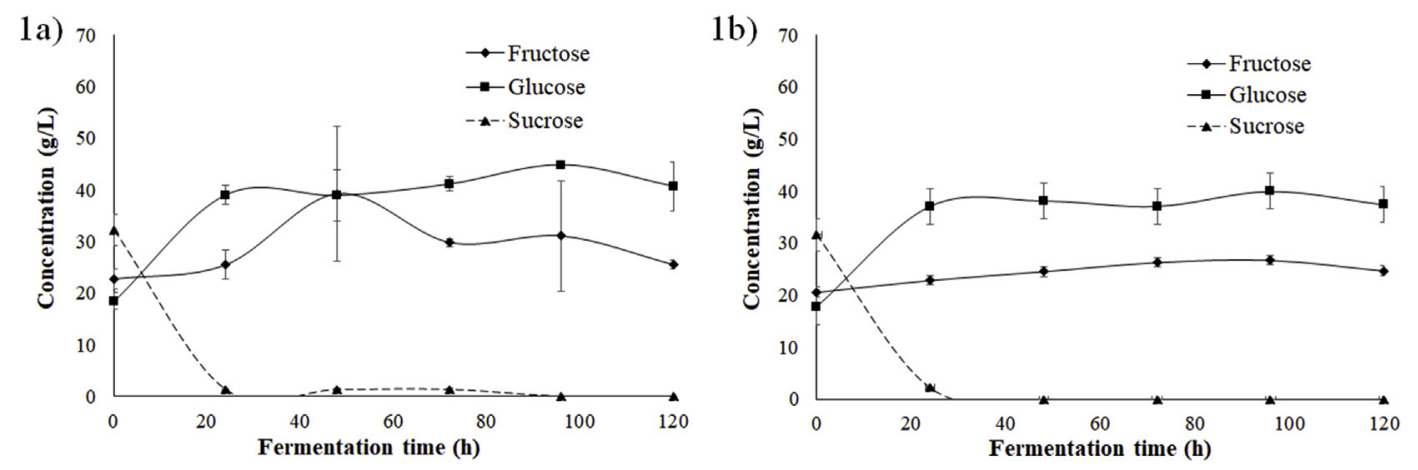

Fig. 1. Sucrose uptake and sugars behavior using A. niger GH1 (a) and A. niger PSH (b).

generation of monosaccharides by competitive inhibitor such as glucose halts the process of FOS synthesis. In addition, aguamiel sucrose concentration (32-34 g/L) was low for the formation of FOS and therefore their production was not possible. High concentrations of sucrose $(500-850 \mathrm{~g} / \mathrm{L})$ are required for FOS production. Hidaka, Hirayama, and Sumi (1988) studied the production of FOS and the enzyme with transfructosylating activity from diverse microorganisms, where Aspergillus niger ATCC 20611 was selected as the most suitable microorganism for FOS production. This is interesting because in the present study Aspergillus niger GH1 did not produce FOS under the tested conditions, therefore, differences among them is probably because of the different concentrations of sucrose used in both studies.

The FOS production with Aspergillus oryzae DIA-MF using aguamiel as culture medium was possible with a maximum yield $(9.24 \mathrm{~g} / \mathrm{L})$ after $24 \mathrm{~h}$ of fermentation this is in accordance with the decrease of sucrose in this same time (Fig. 2a-b). A depletion of sucrose occurred after $48 \mathrm{~h}$ resulting in formation of 1-nystose $\left(\mathrm{GF}_{3}\right)$ followed by 1 -fructofuranosylnystose $\left(\mathrm{GF}_{4}\right)$, while 1-kestose $\left(\mathrm{GF}_{2}\right)$ was formed during the first hours reaching a maximum point at $24 \mathrm{~h}$. According to Prata, Mussatto, Rodrigues, and Texeira (2010) the biosynthesis of these oligomers always is in the order $\mathrm{GF}_{2}<\mathrm{GF}_{3}<\mathrm{GF}_{4}$ from the sucrose as initial molecule. This behavior is possibility because of FOS also can be used as acceptor molecules during the transfructosylation activities. Total FOS production by A. oryzae DIA-MF was $9.24 \mathrm{~g} / \mathrm{L}$ with a yield of product/ total substrate $\left(\mathrm{Y}_{\mathrm{P} / \mathrm{St}}=0.30 \mathrm{~g} / \mathrm{g}\right)$ and product/consumed substrate $(\mathrm{Y}$

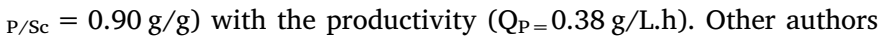
such as Ganaie et al. (2013) reported a higher yield of FOS (19-63\% w/ w) with Aspergillus flavus SI (NFCCI 2364), Aspergillus terreus (NFCCI 2347), Aspergillus niger (ATCC 2601) and Penicillum chrysogenum (MTCC 161) strains at different fermentation times (48-120 h). However, this is first report that use aguamiel of maguey as cultivation medium for FOS production at $24 \mathrm{~h}$. Therefore, in this work, the FOS production by Aspergillus oryzae DIA-MF was improved.

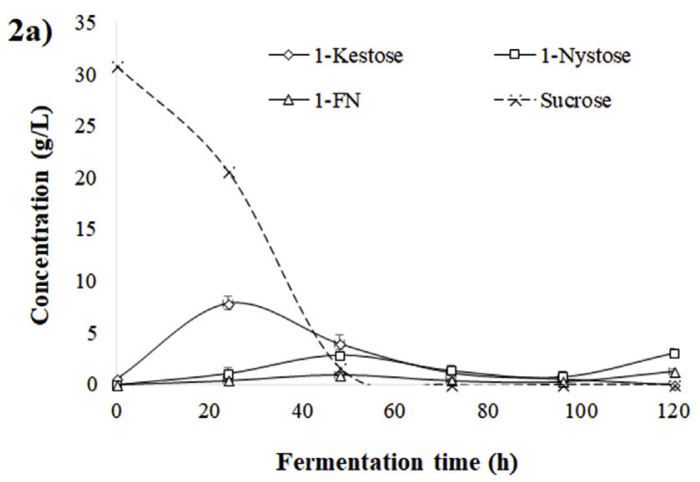

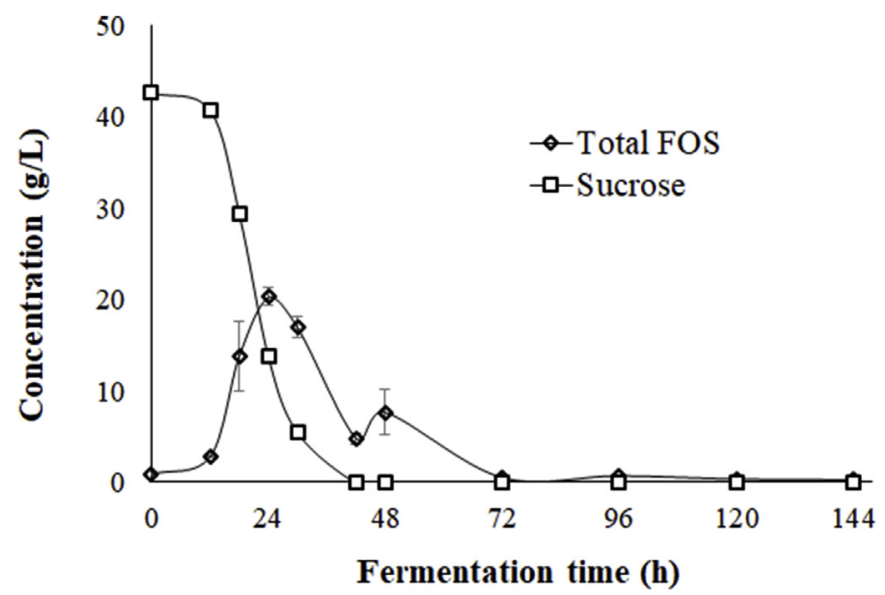

Fig. 3. Maximization of FOS production by A. oryzae DIA-MF using aguamiel as culture medium.

\subsection{Maximization of FOS by Aspergillus oryzae}

A maximization of FOS by Aspergillus oryzae was carried out. In Fig. 3 is shown that FOS production increased when the initial concentration of sucrose was higher $(42.64 \mathrm{~g} / \mathrm{L})$. Oligomers biosynthesis occurs between the first hours, reaching the maximum generation of FOS at $24 \mathrm{~h}$ of fermentation with a total yield of FOS of $20.30 \mathrm{~g} / \mathrm{L}$. The yield of product/total substrate was $\left(\mathrm{Y}_{\mathrm{P} / \mathrm{St}}=0.47 \mathrm{~g} / \mathrm{g}\right)$ and product/ consumed substrate ( $\mathrm{Y}_{\mathrm{P} / \mathrm{Sc}}=0.70 \mathrm{~g} / \mathrm{g}$ ) with the productivity $\left(Q_{P}=0.84 \mathrm{~g} / \mathrm{L} . \mathrm{h}\right)$. Total consumption of sucrose was at $42 \mathrm{~h}$ of fermentation, here also the produced FOS concentration started to decrease. This is possibility because that the microorganism used the FOS as carbon source when sucrose was consumed. Prata et al. (2010) evaluated the FOS production by Penicillium expansum using $200 \mathrm{~g} / \mathrm{L}$ of sucrose and after $36 \mathrm{~h}$ the microorganism started consuming the

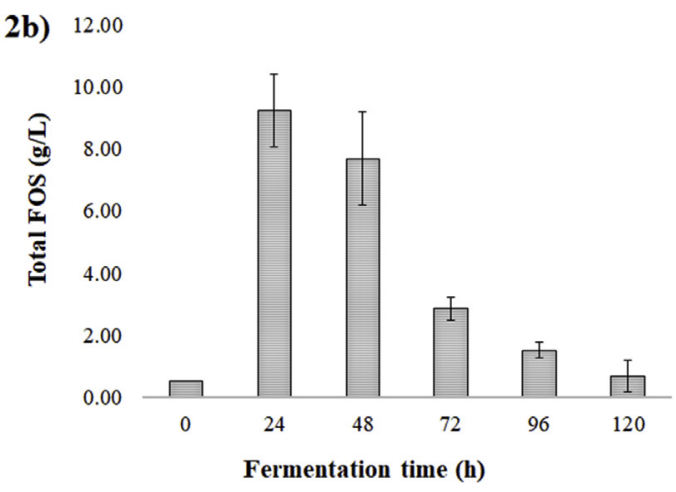

Fig. 2. Sucrose uptake and FOS formation by A. oryzae DIA-MF (a) and total FOS produced at different fermentation times (b). 


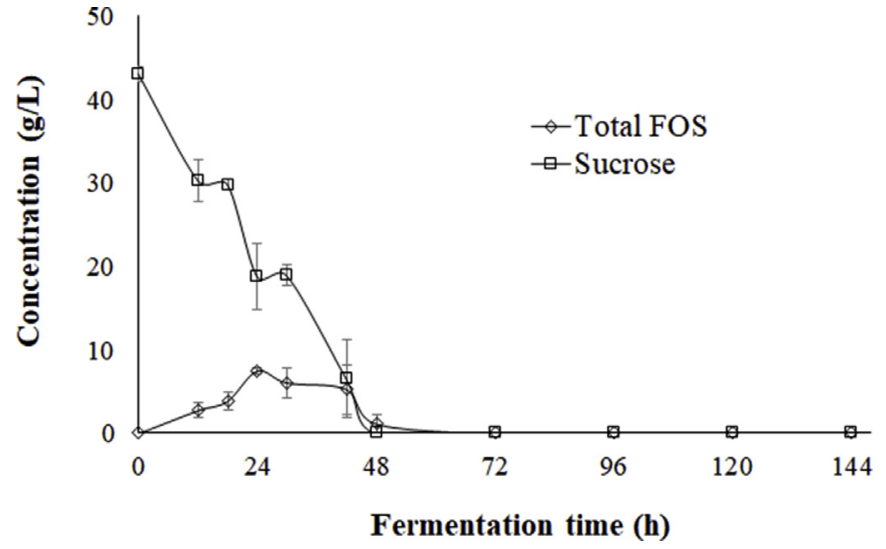

Fig. 4. FOS production by A. oryzae DIA-MF using synthetic Czapek-Dox medium.

produced FOS. Probably, with higher levels of sucrose, FOS production had improved, however, the aim this work was use only the sucrose present in a natural form in the aguamiel which was used as inductor source for prebiotics production. It is necessary to mention that aguamiel was classified in batches and therefore the concentration of sugars was variable. Sánchez, Rodriguez, Silva, and Caicedo (2010), studied the biotransformation of sucrose to fructooligosaccharides and the best conditions of fructooligosaccharides (FOS) production were at sucrose concentrations higher than $50 \% \mathrm{w} / \mathrm{v}$ although they used a fructosyltransferase enzyme of Aspergillus sp. N74.

\subsection{Comparison of FOS production with Czapek-Dox medium}

In this study, we formulate a synthetic medium based in the initial concentrations of fructose, glucose and especially of sucrose $(42.64 \mathrm{~g} / \mathrm{L})$ at the previously established fermentation conditions with aguamiel. As can be noted Fig. 4 shows the FOS production by Aspergillus oryzae DIAMF using Czapek-Dox medium. Where the maximum production of FOS also was at $24 \mathrm{~h}$ with a concentration of $7.42 \mathrm{~g} / \mathrm{L}$ and a total depletion of sucrose was also at $42 \mathrm{~h}$ of fermentation. However, when aguamiel was used as fermentation medium; high concentrations of FOS were produced $(20.30 \mathrm{~g} / \mathrm{L})$. While when Czapek-Dox medium was used, low

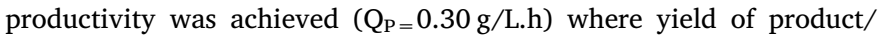
total substrate was $\left(\mathrm{Y}_{\mathrm{P} / \mathrm{St}}=0.17 \mathrm{~g} / \mathrm{g}\right)$ and product/consumed substrate $\left(\mathrm{Y}_{\mathrm{P} / \mathrm{Sc}}=0.31 \mathrm{~g} / \mathrm{g}\right.$ ). Similar behavior was observed in both cases, but the total yield of FOS with aguamiel was two-fold higher that the obtained with the synthetic medium (Fig. 5). Probably, the aguamiel favors production of FOS because it is a nutrient rich environment (Ortiz et al., 2008). Using this by-product for FOS biosynthesis represents a good alternative due to that is an economic material that could be used in industrial scale, because the fermentation process is using very short

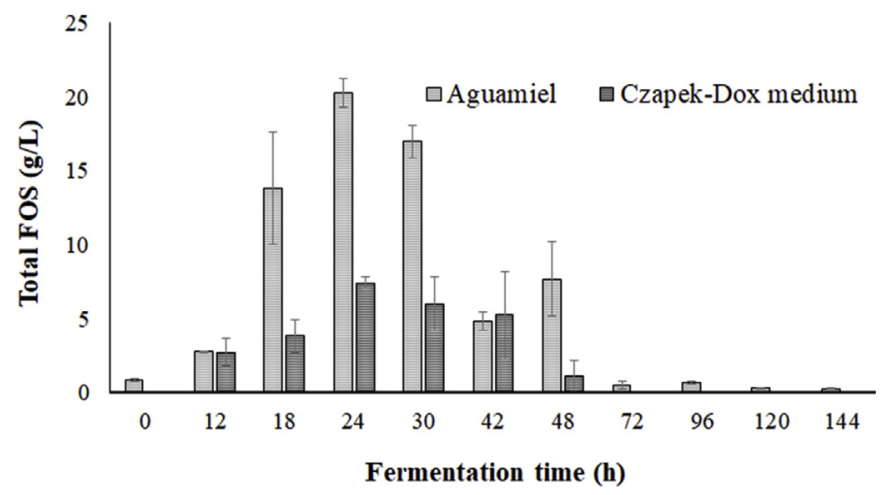

Fig. 5. Comparative FOS production in aguamiel and Czapek-Dox medium.
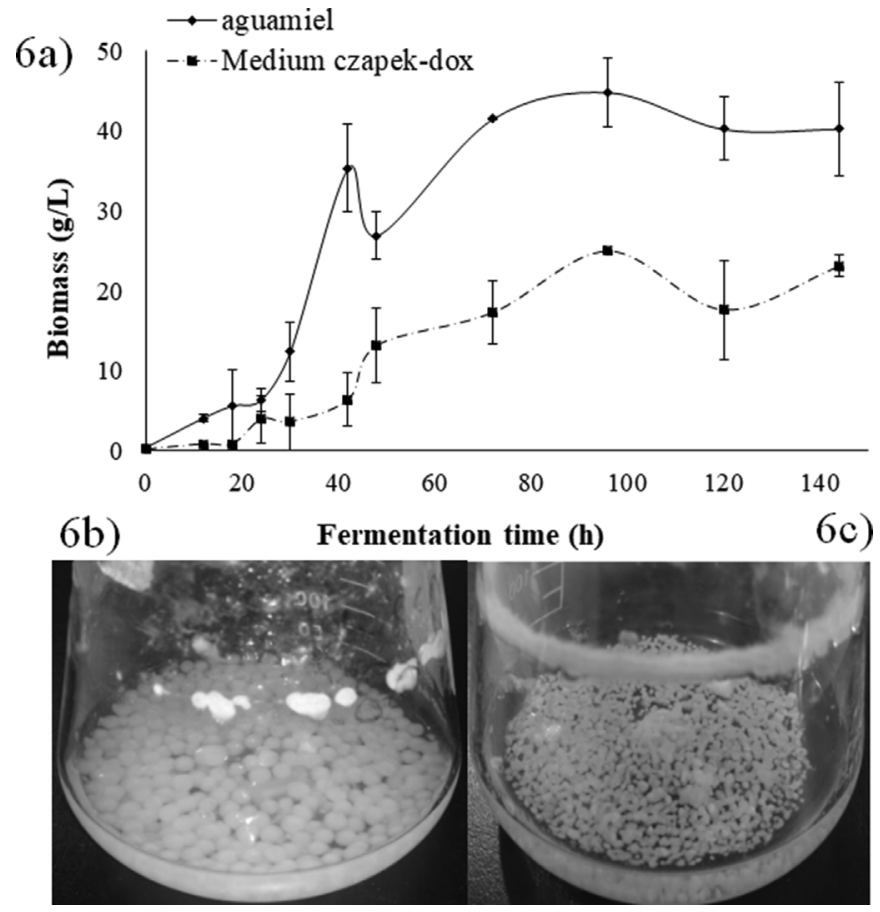

Fig. 6. Kinetic behavior of the biomass production by A. oryzae DIA-MF with aguamiel and Czapek-Dox medium (a), morphology of pellets formed with aguamiel (b) and synthetic Czapek-Dox medium (c).

times $(24-30 \mathrm{~h})$. Another important aspect to be detached is that there is no necessity of adding nutrients for supplementation of this medium and therefore which is a very important economical aspect.

\subsection{Biomass concentration}

Biomass concentration is an important variable during fermentation processes. Fig. 6a shows the biomass production by A. oryzae using aguamiel and medium Czapek-Dox. The results show that the maximum production of FOS was in the microbial exponential phase indicating that the fungus produces FOS as a defensive mechanisms because an osmotic stress in a sucrose rich medium. In addition, sucrose is hydrolyzed to fructose and glucose higher osmotic pressure are generate in the environment (Yoshikawa, Amachi, Shinoyama, \& Fujii, 2006). Biomass production was $44 \mathrm{~g} / \mathrm{L}$ with aguamiel and $24 \mathrm{~g} / \mathrm{L}$ with CzapekDox medium at $96 \mathrm{~h}$ of fermentation (Fig. 6a). The formation of fungal spherical pellets was observed at $24 \mathrm{~h}$ and during the fermentation the pellets grew larges in size with a more pronounced extension diameter using aguamiel as culture medium (Fig. $6 \mathrm{~b}$ and c).

\section{Conclusions}

A high FOS production was obtained when Aspergillus oryzae DIAMF under submerged culture used aguamiel as a culture medium. Therefore, this fungus has the needed enzymes to biosynthesize the FOS, while in this study, the Aspergillus niger GH1 and PSH showed only hydrolytic activity over the sucrose. The maximum yield of FOS by $A$. oryzae DIA-MF was when the sucrose concentrations in aguamiel increased above $40 \mathrm{~g} / \mathrm{L}$ at the $24 \mathrm{~h}$ of fermentation. The composition of total FOS in $\mathrm{g} / \mathrm{L}$ was 1-kestose $>1$-nystose $>1$-fructofuranosylnystose respectively. Important aspects for the FOS production at industrial level should be considered, such as yields, production costs and simplicity in operation, therefore, this study proposes the use of aguamiel as culture medium, because it is a good alternative for the FOS production at low cost and this is the first work focused on the use of an aguamiel for these purposes. 


\section{Acknowledgements}

Authors thank all technical support and comments made by Dra. Adriana C. Flores-Gallegos and Dra. Abril Flores-Maltos. Authors thank National Council for Science and Technology (CONACYT) of Mexico by the financial support through the project No. CB-2011-C01-167764. Author D. B. Muñiz-Márquez also thank CONACYT for the financial support during her postgraduate program (Doctorate) in Food Science and Technology offered by the University Autonomous of Coahuila, Mexico.

\section{Appendix A. Supplementary data}

Supplementary data to this article can be found online at https:// doi.org/10.1016/j.lwt.2018.12.020.

\section{References}

Batista, M., Simões, K., Barros, Pessoni, C., Braga, R. M., \& Ribeiro, R. P. (2013). Production of 6-kestose by the filamentous fungus Gliocladium virens as affected by sucrose concentration. Micoscience, 54, 198-205.

Castro, C. C., Nobre, C., Duprez, M. E., De Weirelda, G., \& Hantsona, A. L. (2017). Screening and selection of potential carriers to immobilize Aureobasidium pullulans cells for fructo-oligosaccharides production. Biochemical Engineering Journal, 118, 82-90.

Ganaie, M. A., Gupta, U. S., \& Kango, N. (2013). Screening of biocatalysts for biotransformation of sucrose to fructooligosaccharides. Journal of Molecular Catalysis B: Enzymatic, 97, 12-17.

Hernandez-López, Z., Rangel-Vargas, E., Castro-Rosas, J., Gómez-Aldapa, C. A., CadenaRamírez, A., Acevedo-Sandoval, O. A., et al. (2018). Optimization of a spray-drying process for the production of maximally viable microencapsulated Lactobacillus pentosus using a mixture of starch pulque as wall material. LWT Food Science and Technology, 95, 216-222.

Hidaka, H., Hirayama, M., \& Sumi, N. (1988). Fructooligosaccharide-producing enzyme from Aspergillus niger ATCC20611. Agricultural \& Biological Chemistry, 52, 1181-1187. Muñiz, D. B., Contreras, J. C., Rodríguez, R., Mussatto, S. I., Wong-Paz, J. E., Teixeira, J. A., et al. (2015). Influence of thermal effect on sugars composition of Mexican Agave syrup. CyTA - Journal of Food, 13, 607-612.

Nascimento, A. K. C., Nobre, C., Cavalcanti, M. T. H., Teixeira, J. A., \& Porto, A. L. F. (2016). Screening of fungi from the genus Penicillum for production of $\beta$-fructofuranosidase and enzymatic synthesis of fructooligosaccharides. Journal of Molecular Catalysis B: Enzymatic, 134, 70-78.

Nobre, C., Alves Filho, E. G., Fernandes, F. A. N., Brito, E. S., Rodrigues, S., Teixeira, J. A. et al. (2018). Production of fructo-oligosaccharides by Aspergillus ibericus and their chemical characterization. LWT Food Science and Technology, 89, 58-64.

Ortiz, R. I., Pourcelly, G., Doco, T., Williams, P., Dornier, M., \& Belleville, M. P. (2008), Analysis of the main components of the aguamiel produced by the maguey-pulquero (Agave mapisaga) throughout the harvest period. Journal of Agricultural and Food Chemistry, 56, 3682-3687.

Prata, M. B., Mussatto, S. I., Rodrigues, L. R., \& Texeira, J. A. (2010). Fructooligosaccharide production by Penicillium expansum. Biotechnology Letters, 32, 837-840.

Sánchez, O. F., Rodriguez, A. M., Silva, E., \& Caicedo, L. A. (2010). Sucrose biotransformation to fructooligosaccharides by Aspergillus sp. N74 free cells. Food and Bioprocess Technology, 3, 662-673.

Won, J. (1996). Fructooligosaccharides-Occurrence, preparation and application. Enzyme and Microbial Technology, 19, 107-117.

Xie, Y., Zhou, H., Lui, C., Zhang, J., Li, N., Zhao, Z., et al. (2017). A molasses habitat derived fungus Aspergillus tubingensis XG21 with high $\beta$-fructofuranosidase activity and its potential use for fructooligosaccharides production. AMB Express, 7, 128.

Yoshikawa, J., Amachi, S., Shinoyama, H., \& Fujii, T. (2006). Multiple $\beta$-fructofuranosidases by Aerobasidium pollulans DSM2404 and their roles in fructooligosaccharides production. FEMS Microbiology Letters, 265, 159-163.

Zhang, J., Liu, C., Xie, Y., Li, N., Ning, Z., Du, N., et al. (2017). Enhancing fructooligosaccharides production by genetic improvement of the industrial fungus Aspergillus niger ATCC 20611. Journal of Biotechnology, 249, 25-33. 